\title{
Finite Projective Spaces, Geometric Spreads of Lines and Multi-Qubits
}

\author{
Metod Saniga \\ Astronomical Institute, Slovak Academy of Sciences \\ SK-05960 Tatranská Lomnica, Slovak Republic
}

(6 July 2012)

\begin{abstract}
Given a $(2 N-1)$-dimensional projective space over $\mathrm{GF}(2), \mathrm{PG}(2 N-1,2)$, and its geometric spread of lines, there exists a remarkable mapping of this space onto $\mathrm{PG}(N-1,4)$ where the lines of the spread correspond to the points and subspaces spanned by pairs of lines to the lines of $\mathrm{PG}(N-1,4)$. Under such mapping, a non-degenerate quadric surface of the former space has for its image a non-singular Hermitian variety in the latter space, this quadric being hyperbolic or elliptic in dependence on $N$ being even or odd, respectively. We employ this property to show that generalized Pauli groups of $N$-qubits also form two distinct families according to the parity of $N$ and to put the role of symmetric Pauli operators into a new perspective. The $N=4$ case is taken to illustrate the issue, due to its link with the so-called black-hole/qubit correspondence.
\end{abstract}

MSC Codes: 51Exx, 81R99

PACS Numbers: 02.10.Ox, 02.40.Dr, 03.65.Ca

Keywords: Finite Projective Spaces; Spreads of Lines; Pauli Groups of $N$-Qubits

Multiple qubit states play a key role in various fields of quantum information theory like quantum computing, coding and quantum error-correction (see, e.g., 1]). Recently, and rather surprisingly, they have also been recognized to be of great relevance for getting insights into the nature of entropy formulas of a certain class of stringy black hole solutions (see, e. g., [2]). It is, therefore, important to deepen our understanding of these fundamental buildings blocks of quantum world. In the present note we do so through the geometry of their associated generalized Pauli groups.

Let $\mathrm{PG}(d, q)$ be a $d$-dimensional projective space over $\mathrm{GF}(q), q$ being a power of a prime 1 A $t$-spread $\mathcal{S}$ of $\operatorname{PG}(d, q)$ is a set of $t$-dimensional subspaces of $\operatorname{PG}(d, q)$ which partitions its point-set [4]. If the elements of $\mathcal{S}$ in a subspace $V$ form a $t$-spread on $V$, one says that $\mathcal{S}$ induces a $t$-spread on $V$. A $t$-spread $\mathcal{S}$ is called geometric (or normal) if it induces a $t$-spread on each $(2 t+1)$-dimensional subspaces of $\mathrm{PG}(d, q)$ spanned by a pair of its elements. It is a well-known fact that $\operatorname{PG}(d, q)$ possesses a $t$-spread iff $(t+1) \mid(d+1)$; moreover, this condition is also sufficient for $\operatorname{PG}(d, q)$ to have a geometric $t$-spread. B. Segre showed [5] that a geometric $t$-spread of $\mathrm{PG}(N(t+1)-1, q), N \geq 2$, gives rise to a projective space $\mathrm{PG}\left(N-1, q^{t+1}\right)$ as follows: the points of this space are the elements of $\mathcal{S}$ and its lines are the $(2 t+1)$-dimensional subspaces spanned by any two distinct elements of $\mathcal{S}$, with incidence inherited from $\mathrm{PG}(N(t+1)-1, q)$. For a particular case of $t=1$ (i. e., a spread of lines), Dye [6] demonstrated that a hyperbolic or an elliptic quadric of $\mathrm{PG}(2 N-1, q)$ has an induced (geometric) spread of lines if and only if $N$ is, respectively, even or odd, in which case it is mapped onto a non-singular Hermitian variety $\mathrm{H}\left(N-1, q^{2}\right)$ of $\mathrm{PG}\left(N-1, q^{2}\right)$. We shall now show that this property has for $q=2$ a very interesting physical implication.

It is already a firmly established fact $[7,8,9,10$, that the commutation relations between the elements of the generalized Pauli group of $N$-qubits, $N \geq 2$, can be completely reformulated in the geometrical language of symplectic polar space of rank $N$ and order two, $\mathrm{W}(2 N-1,2)$; the generalized Pauli operators (discarding the identity) answer to the

\footnotetext{
${ }^{1}$ For the standard mathematical nomenclature and notation employed in what follows, see, e. g., 3 .
} 
points of $\mathrm{W}(2 N-1,2)$, a maximally commuting subset has its representative in a maximal totally isotropic subspace of $\mathrm{W}(2 N-1,2)$ and commuting translates into collinear. One of the most natural representations of $\mathrm{W}(2 N-1,2)$ is that in terms of the points and the set of totally isotropic subspaces of $\mathrm{PG}(2 N-1,2)$ endowed with a symplectic polarity. Employing this representation, it has been found in 8 that in the real case the symmetric elements/operators of the $N$-qubit Pauli group always lie on a hyperbolic quadric in the ambient space PG $(2 N-1,2)$. Combining this fact with Dye's result, we arrive at our main observation: it is only for $N$ even when all symmetric generalized Pauli operators of $W(2 N-1,2)$ can be mapped to the points of an Hermitian variety of the space $P G(N-1,4)$ associated through a geometric spread of lines with the ambient space $P G(2 N-1,2)$. Hence, in this regard, when it comes to generalized Pauli groups 'even-numbered' multi-qubits are found to stand on a slightly different footing than 'odd-numbered' ones.

We shall finish this communication by briefly mentioning an especially interesting even case, $N=4$. Here, a hyperbolic quadric $\mathrm{Q}^{+}(7,2)$ of $\mathrm{PG}(7,2)$ formed by the symmetric operators is well known for its puzzling triality swapping points and two systems of generators and has for its spread-induced image an Hermitian surface $\mathrm{H}(3,4)$ of $\mathrm{PG}(3,4)$ (see, e.g., 11]). This Hermitian surface is, in turn, nothing but the generalized quadrangle GQ $(4,2)$ in disguise (see, e.g., [12]), the dual of which - $\operatorname{GQ}(2,4)$ - was found to play a prominent role in the so-called black-hole-qubit correspondence, by fully encoding the $E_{6(6)}$ symmetric entropy formula describing black holes and black strings in $D=5$ [13]. Our finding thus, inter alia, not only opens up an unexpected window through which also four-qubit Pauli group, like its lower rank cousins, could find its way into some black hole entropy formula(s), but also puts the role of symmetric operators into a new perspective. It is also important to keep in mind this remarkable three-to-one correspondence, i.e., that it is always a triple of (collinear) operators of the ambient space $\mathrm{PG}(7,2)$ which comprises a single point of $\mathrm{PG}(3,4)$.

\section{Acknowledgements}

This work was partially supported by the VEGA grant agency, projects Nos. 2/0092/09 and 2/0098/10. The idea exposed in this paper originated from discussions with Prof. Hans Havlicek, Dr. Boris Odehnal (Vienna University of Technology) and Dr. Petr Pracna (J. Heyrovský Institute of Physical Chemistry, Prague).

\section{References}

[1] M. A. Nielsen and I. L. Chuang: Quantum Computation and Quantum Information, Cambridge University Press, Cambridge 2000.

[2] L. Borsten, D. Dahanayake, M. J. Duff, H. Ebrahim and W. Rubens: Phys Reports 471, 113 (2009).

[3] J. W. P. Hirschfeld and J. A. Thas: General Galois Geometries, Oxford University Press, Oxford 1991.

[4] J. Eisfeld and M. Storme: (Partial) $t$-spreads and minimal $t$-covers in finite projective spaces, lecture notes available from the web site http://cage.rug.ac.be/ fdc/intensivecourse2/storme2.ps.

[5] B. Segre: Ann. Mat. Pura Appl. 64, 1 (1964).

[6] R. H. Dye: J. London Math. Soc. 33, 279 (1986).

[7] M. Saniga and M. Planat: Adv. Studies Theor. Phys. 1, 1 (2007).

[8] H. Havlicek, B. Odehnal and M. Saniga: SIGMA 5, Art. 096 (2009).

[9] K. Thas: EPL 86, Art. 60005 (2009).

[10] P. Vrana and P. Lévay: J. Phys. A: Math. Theor. 43, Art. 125303 (2010).

[11] H. Havlicek, B. Odehnal and M. Saniga: Des. Codes Crypt. 62, 343 (2012).

[12] S. E. Payne and J. A. Thas: Finite Generalized Quadrangles, Pitman, Boston-LondonMelbourne 1984. 
[13] P. Lévay, M. Saniga, P. Vrana and P. Pracna: Phys Rev D 79, Art. 084036 (2009). 